\title{
A Survey on Secure Routing Protocols for Mobile Ad-hoc Network
}

\author{
Zalte S.S. ${ }^{\# 1}$,Ghorpade V.R." \\ \#1 Shivaji University,Kolhaur,9422785209 \\ \#2 Bharati Vidyapeeth,College of Engineering
}

\section{ABSTRACT}

Mobile Ad-hoc network is instant ,autonomous and easily assembled in havoc terrain, where pre-deployment of infrastructure is impossible. On other hand, it is sometimes notorious because of its unique characteristics such as imprudent topology, promiscuous mode, frequent link breakage and resource constraints. There is extraordinary gap between MANET's nature and security in applications. Secure routing in MANET becomes excellent topic now a day. If security not properly impose consequences will be resource consumption and degradation of network performance. This paper gives survey on secure routing protocols for MANET.

Key words: Mobile Ad-hoc Network, resource constraint, security, routing protocols, network performance.

\section{Corresponding Author: Zalte S.S.}

\section{INTRODUCTION}

Mobile Ad-hoc Network is collection of mobile devices which are easily assembled in havoc terrain temporarily, where pre-deployment of infrastructure impossible. It is cheap, instant and wireless network as shown in Fig 1.[1]

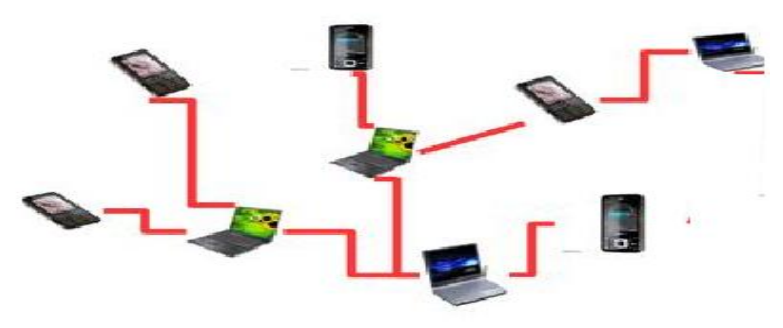

Fig 1:Mobile Ad-hoc Network

MANET has some unique characteristics like imprudent topology, promiscuous mode, frequent link breakage and resource constraints, so routing in MANET is different from traditional routing. To build intrinsic mutual trust between nodes are difficult because nodes are not at fixed position, they are movable. This type of volatile topology leads to detecting malicious nodes troublesome. Security applied for wired network is not worked for MANET. 
DOI: https://dx.doi.org/10.26808/rs.ca.i8v1.02

\section{Attacks on MANET}

MANET is suffering from various attacks such as active attacks and passive attacks.[2]

\section{Passive Attacks}

Passive attacks are difficult to detect because they does not disrupt routine operations of network. passive attackers only monitor data traffic and snoop the data without modifying. Here Confidentiality to data is violated. There are some passive attacks.

- Traffic Monitoring: There are so many networks like satellite, WLAN and cellular are also suffering from such attacks. By monitoring data traffic attackers capture sensitive information for its personnel use.

- Eavesdropping: malicious node can overhear the data transmission. Transmitted message can be eavesdropped and fraud message can be injected into network.

- Traffic Analysis: Traffic analysis is a passive attack used to gain information on which nodes communicate with each other and how much data is processed.

- Syn flooding: By sending repeatedly RREQ, it floods network and make unavailability of resources such as bandwidth, memory and energy.

\section{Active attacks}

Active attacks are easy to detect than prevent. Active attacks disturb whole network routine. passive attacks are difficult to detect because it does not disturb network routine. Active attacks can be imposed by internal attackers or external attackers.

- External attacks are carried out by the nodes which are not part of network. Internal attackers are one of the attacker among the participant which are compromised,so hard to detect.

- Black hole Attack: In this attack, an attacker advertises it has shortest path to destination or he is a destination. Attacker also send a zero metric for all destination which causes it attract all traffic.

- Wormhole Attack: - In a wormhole attack, with the help of co-ordination between malicious nodes attackers forcefully tunnel the packet from one end to another. This tunnel between two colluding attacks.[5]

- Replay attack: An attacker captures previous session and resend it again repeatedly to consume network resources.

- Sinkhole: In a sinkhole attack, a malicious node advertise it has greatest sequence number and smallest hop count path to attract traffic from all neighboring nodes.

- Spoofing Attack:-In spoofing attack, the attacker uses valid identity of another node; hence it receives messages meant for that node. 
DOI: https://dx.doi.org/10.26808/rs.ca.i8v1.02

- Sybil attack: The Sybil attack aims at distributed system environments. The attacker tries to act as several different identities/nodes rather than one.

- Man-in-the-middle attack: An attacker situated middle between the sender and receiver and captures data packet which being sent to destination.

- Byzantine attack: A attacker with the help of other neighboring node carry out attacks such as routing loops, forwarding packets through non-optimal paths or selectively dropping packets which results in disruption or degradation network performance.

- Flooding-Malicious nodes may flood the network by injecting false packets. Due to flooding legitimate nodes may starved network resources like bandwidth, battery and energy [3],[4] etc.

- Rushing-In rushing attack, attacker captures route request packet and transmit to its neighboring node with high speed. When destination node received route request packet it discards other route request packets which are coming from legitimate nodes.[6]

\section{LITERATURE SURVEY}

In this paper, we explore some of the existing security mechanism against malicious attacks in MANETs and also the intrusion detection techniques to detect them. First, we have provided the taxonomy of attacks against MANETs. Then, we primarily focused on how the network layer attacks are carried out against MANET.

Author proposed a secure routing protocol (SRP) in [7]. This protocol is based on a pre-shared key between source and destination nodes. A source node produces RREQ message and broadcasts it among its neighbors. When destination node receives RREQ message it verifies the source node and establish the route.

Drawback of SRP is that it does not authenticate intermediate nodes, it is vulnerable to attacks such as fabricated route error messages.

$\operatorname{In}[8]$ author secured on demand routing protocol ,Dynamic Source Routing. In this protocol not only source and destination but also intermediate nodes are authenticated. The main task of this protocol is to allow intermediate nodes to authenticate its predecessor node and also detect attacks like modification, fabrication and fake RREQ. Finally, destination node authenticate all nodes in the route. This protocol provides security against reply attack, rushing attack, IP spoofing and man in the middle attack. Protocol is based on asymmetric cryptography, so it consumes more battery due to lot of calculation.

Same author proposed key management in[9] which combines symmetric cryptography for secure communication as well as a asymmetric cryptography for authenticate other nodes and share session keys. In this proposed method a group leader can generate, distribute, update and revoke keys in its group and a provable secure routing protocol. The main objective is that it authenticate source, destination and intermediate nodes in the list of RREQ message. This protocol provides security against various attacks such as rushing, replay and man-in-middle attack 
In this protocol malicious node is detected by detecting its malicious behavior in [10] such as packet dropping, fake advertising, misrouting, violating security rules. This can be detected and defended by using key management mechanism. Here packet is sent by secrete key and it is done with encryption and decryption. In this author used two scheme public key. In the dynamic key management scheme, each node has a pair of private/public key issued by public Key Infrastructure or certificate authority (CA) and RSA to generate session keys. It is used to authenticate nodes.

Author proposed DSR protocol in [11] which is based on aggregate signatures (merge several signatures in one). For public key cryptography author has been used elliptic curve cryptography.

Drawback is unable to detect node who has not signed in case of negative result.

Author used reputation based system which is based on node reputation and route reputation to detect malicious and selfish nodes in [12]. Author calculate these using contribution, Capability of Forwarding $(\mathrm{CoF})$ To implement this scheme author have used dsr protocol. Author also handle hot point issue where one node carry lot traffic load, it distribute load to alternative path and avoid hot points. It fight against passive attacks. Advantage of COSR is that it is capable of avoiding hot points.

To improve performance and security author have used in [13].The security of AODV will be based on one-way hash, two-way hash and digital signature. Here author generates two signatures . Intermediate nodes verify only first signature and accept packet and destination node verify second signature to check authenticity and integrity.

Advantages no certificate and key management scheme, less overhead of calculation, less battery consumption.

Author proposed modified form of AODV in [14] that is secured routing protocol (SecAODV), All nodes within MANET are distributed a certain IPv6 address value. In this scheme, the secured communication channel is established in between source and destination node depending upon the idea of Statistically Unique and Cryptographically Verifiable (SUCV) who confirms secured and secret binding between IPv6 address and key node.

STAND protocol proposed in [16]. It finds out wormhole attack in static and mobile environment. It also finds out correct and incorrect neighbors in the network. To verify the neighbors of a given node, our protocol reconstructs the topology of its neighborhood by applying Multidimensional Scaling (MDS) to the available pair wise distances. MDS (multidimensional scaling) is visualization technique used to visualize set of typically high dimensional object in lower dimensional node.

In [17] advanced encryption (AES) and elliptical curve cryptography (ECC) are combined for encryption of node. XORDUALRSA algorithm is considered for authentication and its robustness, (MD5) for integrity. The splitting of text increases protocol's strength. Plain text is encrypted by AES and secret key encrypted by ECC. The proposed work is suitable for time reduction and power saving. 
DOI: https://dx.doi.org/10.26808/rs.ca.i8v1.02

In[18] Here author proposed S-DSR protocol which is based on trusted information of neighboring nodes. By collecting locality information and trust information about nodes ,probability of the packets which are repeatedly sent along the same path can be reduced. Author also have peer to peer based approach .To check peers trustworthiness. nodes collect recommendation from all other peers and calculate reputation value. The peer having higher reputation value selected as best peer.

This protocol reduces collision and low overhead during the route discovery. It also increases pdr and throughput.

In [19] author provided double shielded security to routing message and data packets in AODV. Data packets are secured by generating unique secret key by using sha1 algorithm. It provides a unique session key by using diffi-helman signature for each route to secure the data communication. SWR protocol used to encrypt route message by using symmetric cryptography sha1 and asymmetric cryptography by using node's public key. Some Attacks are resolved like route cache poisoning, no participation in discovery process, modification, packet drop.

Author proposed Secure Optimal Reactive Routing Protocol (SORRP) in [20] for mobile ad-hoc networks. The proposed protocol clubs the pros of both asymmetric and symmetric key cryptography and provides end-to-end authentication, message integrity, non-repudiation and confidentiality. While sharing secrete key it is protected with asymmetric cryptography. It also clubs the best characteristics of both reactive and proactive routing protocol. Like any other reactive routing protocol it determines the route on demand, thus eliminating the overhead involved in identifying all possible routes beforehand. SORRP maintain a list containing all the neighbors, and their connecting cost. This is used in determining the shortest route. This cost calculated by taking the difference between timestamp of sending and receiving hello messages.

This thesis[21] is based on historical information about node stored in small table. History based route selection increases packet delivery ratio and improved stability. Packet forwarding done through only with qualitative nodes this quality judge by monitoring how many packets are forwarded and how many packets are dropped.

\section{CONCLUSION}

Indeed, security is vital concern in variety of applications which are based on MANET. There are lots of threats and vulnerabilities already present in existing literature. So many researchers proposed various types of security mechanisms which are included in this survey paper but no one provide enough security and privacy from the start. Future research should be focused on designing components of secure routing protocol provisioning so that they would be more suitable for MANET.

Furthermore, in case of cross-layer attacks combination of different security mechanism need to enforced. This is worth exploring in future to make MANETs more secure and reliable.

In future there is need to study various security mechanism with its pros and cons and to develop security routing protocol which able to provide security to route as well as to data packets.

\section{REFERENCE}

[1] A.Aarti, Dr. S. S. Tyagi, 2013. Study of MANET: Characteristics, Challenges, Application and Security Attacks, IJARCSSE. 
DOI: https://dx.doi.org/10.26808/rs.ca.i8v1.02 International Journal of Computer Application (2250-1797) Issue 8 Volume 1, January- February 2018

[2] Mohammad Wazid, Rajesh Kumar Singh, R. H. Goudar, 2011. A Survey of Attacks Happened at Different Layers of Mobile Ad-Hoc Network \& Some Available Detection Techniques, (IJCA).

[3] http://www.slideshare.net/sunitasahu101/attacks-in-manet

[4] Gagandeep, Aashima, Pawan Kumar,June 2012.Analysis of Different Security Attacks in MANETs on Protocol Stack A-Review International Journal of Engineering and Advanced Technology (IJEAT) ISSN: 2249 - 8958, Vol.1, No.5

[5] Jyoti Raju and J.J. Garcia-Luna-Aceves," A comparison of On-Demand and TableDriven Routing for Ad Hoc Wireless Networks", in Proceeding of IEEE ICC, June 2000

[6] Satyam Shrivastava," Rushing Attack and its Prevention Techniques", IJAIEM, Volume 2, Issue4, April 2013,pp-453-456

[7] P. Papadimitratos, and Z. Haas, "Secure Routing for Mobile Ad hoc Networks", Proceeding of SCS Communication Networks and Distributed Systems Modeling and Simulation, January, 2002

[8] Kamal Kumar Chauhan1, Amit Kumar Singh Sanger2, Virendra Singh Kushwah3, "Securing On-Demand Source Routing in MANETs", 2010 IEEE,pp-294-297

[9] Kamal Kumar Chauhan1 and Amit Kumar Singh Sanger2, "Securing Mobile Ad hoc Networks: Key Management and Routing", International Journal on AdHoc Networking Systems (IJANS) Vol. 2, No. 2, April 2012,pp-65-75

[10] Bhuvaneswari M, Dinesh naik, "Secure optimal routing protocol in Manets", 2014 International Conference on Control, Instrumentation,Communication and Computational Technologies (ICCICCT) IEEE,pp-1320-1323

[11] José Luis Tornos, Joan Josep Piles and José Luis Salazar, "ADSR: Authenticated DSR",2011 6th International Conference on Risks and Security of Internet and Systems (CRiSIS), 2011 IEEE,pp-1-8

[12] Fei Wang, Yijun Mo, Benxiong Huang, "COSR: Cooperative On-Demand Secure Route Protocol in MANET", ISCIT 2006,2006 IEEE,pp-890-893

[13] Morli Pandya,Ashish Kr. Shrivastava.(2013)," Improvising Performance with Security of AODV Routing Protocol for MANETs", International Journal of Computer Applications. 78, p1- 1-7.

[14] A. Patwardhan, J. Parker, A. Joshi, M. Iorga and T. Karygiannis, "Secure Routing and Intrusion Detection in Ad Hoc Networks, " in Proc. of the 3rd IEEE - International conference on Pervasive Computing and Communications, March, 2005, pp. 191-199.

[15] Jihye Kim, Gene Tsudik, "SRDP: Secure route discovery for dynamic source routing in MANETs", elsevier,Ad Hoc Networks 7 (2009) 1097-1109.

[16] Somayeh Taheri, Radu Stoleru,Dieter Hogrefe, " Secure Neighbor Discovery in Mobile Ad Hoc Networks through ...local topology visualization", 2016 IEEE 30th International Conference on Advanced Information Networking and Applications (AINA),pp- 933940. 
DOI: https://dx.doi.org/10.26808/rs.ca.i8v1.02 International Journal of Computer Application (2250-1797) Issue 8 Volume 1, January- February 2018

[17] Yasmin Alkady, Mohmed I. Habib, Rawya Y. Rizk, " A New Security Protocol Using Hybrid Cryptography Algorithms", 978-1-4799-3370-9/13@2013 IEEE,pp-109-115.

[18] V.Bhargavi, Dr. M.Seetha, S.Viswanadharaju, " A Trust Based Secure Routing Scheme for MANETS",2016 IEEE, pp-565-570.

[19] Jarupula Rajeshwal, Gugulotu Narsimha, " Secure way routing protocol for mobile ad hoc network", Wireless Networks,springer,February 2017, Volume23, Issue 2, pp 345-354

[20] Soma Saha, Rituparna Chaki, Nabendu Chaki, " A New Reactive Secure Routing Protocol for Mobile Ad-Hoc Networks",7th Computer Information Systems and Industrial management Applications,2008 IEEE,PP-103-108.

[21] Peter Michael Cappeto, "History-Based Route Selection for Reactive Adhoc Routing Protocols", thesis, Washington State University,may 2007 